\title{
Effect of Physical Properties of Pr-Nd Oxide on Its Melton Salt Electrolysis
}

\author{
Wenyi Zhao*, Rongrong Fan, Zhaoqiang Li, Yan Wang, Cheng Zhang, Rong Wang, Xin Guo, \\ Yu Wang, Xuxia Zhang
}

Department of Hydrometallurgy, Baotou Research Institute of Rare Earths, Baotou, China

\author{
Email address: \\ 786093386@qq.com (Wenyi Zhao) \\ ${ }^{*}$ Corresponding author
}

\section{To cite this article:}

Wenyi Zhao, Rongrong Fan, Zhaoqiang Li, Yan Wang, Cheng Zhang, Rong Wang, Xin Guo, Yu Wang, Xuxia Zhang. Effect of Physical Properties of Pr-Nd Oxide on Its Melton Salt Electrolysis. International Journal of Materials Science and Applications.

Vol. 7, No. 5, 2018, pp. 186-191. doi: 10.11648/j.ijmsa.20180705.13

Received: August 13, 2018; Accepted: August 28, 2018; Published: October 11, 2018

\begin{abstract}
Industrial Pr-Nd oxide product has different physical properties, and shows different behaviors in the process of producing Pr-Nd metal by molten salt electrolytic. To meet the specific requirements of molten salt electrolysis for the physical properties of Pr-Nd oxide, different physical properties of raw materials such as particle size, specific surface area, morphology, bulk density and mobility were compared by chemical analogy experiment and actual production, the influence of physical properties of Pr-Nd oxide on its electrolysis process were studied, and the physical properties of the suitable electrolytic Pr-Nd oxide are quantified. The results show that particle size and morphology are the most important physical properties of Pr-Nd oxide in the electrolysis process. Pr-Nd oxide with good physical property requires the particle size is uniform and not too big or small. Additionally, the microstructure should not be over dispersed, but with agglomerate status, besides the micro surface should have porous structure to avoid melting phenomenon. The physical properties of raw materials produced by the method of oxalic acid precipitation are more stable than that by the ammonium bicarbonate precipitation method, and the good and stable physical properties are more suitable for molten salt electrolysis. So the oxalic acid precipitation method can be used as reference of the ammonium bicarbonate precipitation method.
\end{abstract}

Keywords: Pr-Nd Oxide, Physical Properties, Electrolysis, Dissolution Rate

\section{Introduction}

Praseodymium and neodymium (Pr-Nd) metal is an important raw material in the production of neodymium iron boron permanent magnetic material, which is mainly produced by Pr-Nd oxide molten salt electrolysis method in a fluoride system. Currently, although electrolysis process is a relative mature process, improvement of $\mathrm{Pr}-\mathrm{Nd}$ oxide performance becomes a key factor in the green smelting and effective utilization of rare earth metals. Both chemical and physical properties of Pr-Nd oxide were studied. The current rare earth industry has a certain chemical standard of the content of impurities in Pr-Nd oxide including carbon, silicon, iron, calcium and chloride ions during electrolytic process, but the effect of its physical properties on electrolysis process are rarely studied. Pr-Nd metal and aluminum are both produced by molten salt electrolysis, in which the working principles of the electrolytic cells are the same. Researches on the influence of physical properties of alumina electrolytic production and improvement of its physical properties to meet the requirements of aluminum electrolysis are plentiful [1-5]. When the physical properties of particle size and fluidity of alumina changed, the situation of aluminum electrolytic cell will also significantly change during the alternation of materials. While it takes some time for the technical conditions to be adjusted to adapt the new material. As a result, the electrical energy consumption will increase. So, the physical properties of alumina should be stable as long as possible. Combining the aluminum electrolysis process, and comparing different rare earth metal producers, it was discovered that industrial Pr-Nd oxide products had different physical properties, and showed different behaviors in electrolytic production [6-12]. Under the circumstances of 
unchanged electrolysis process, even the chemical composition of Pr-Nd oxide is qualified and conforms to the electrolytic standard, the electrolytic cell will still be effected by high viscosity of electrolyte, sticky bottom and serious bottom crust etc., whichthe leads to low efficiency of electrolysis and decreases the yield and quality of the metal.

So far the physical properties of Pr-Nd oxide are limited to some simple indicators such as fluidity, dustless, bottom sinking and so on. Additionally the producers do not have other physical properties' standards of suitable electrolytic Pr-Nd oxide, as a result the Pr-Nd oxide producers don't have a guide to produce products with specific physical properties. Moreover, enterprises cannot reduce the risks of production due to the instability of physical properties of raw materials. Therefore, this study focuses on testing physical properties of industrial Pr-Nd oxide, such as particle size, specific surface area, bulk density, morphology and dissolution properties of Pr-Nd oxide respectively. The influence of physical properties of Pr-Nd oxide on its electrolysis process were analyzed. The physical properties of the suitable electrolytic Pr-Nd oxide were revealed, and the specific requirements of physical properties of Pr-Nd oxide were formulated.

\section{Method}

\subsection{Raw Materials and Reagents}

Raw materials: Industrial Pr-Nd oxide

Acid solution: Hydrochloric acid solution with the concentration of $2 \mathrm{~mol} / \mathrm{L}$.

\subsection{Test of Pr-Nd Oxide's Solubility}

A certain amount of Pr-Nd oxide was placed in a beaker, and then a certain volume of hydrochloric acid solution was added into the beaker rapidly. After a period of time at room temperature, the supernatant was quickly poured out, the total rare earth amount of the supernatant was measured and the acid dissolution rate was calculated.

\subsection{Characterization of Physical Properties of Pr-Nd Oxide}

The morphology and aggregation of the samples were observed by S-3400N scanning electron microscope of Hitachi Corporation. The particle size of samples was tested by LS230 type laser particle size tester of the Coulter. The specific surface area of samples was measured by SA3100 type specific surface area measurement. Bulk density and mobility of samples were tested by self-made devices.

\section{Results and Discussion}

\subsection{Physical Properties of Pr-Nd Oxide}

Pr-Nd oxide is the main raw material for preparation of Pr-Nd metal, and the main preparation technologies are oxalic acid and ammonium bicarbonate precipitation methods. Based on previous practical electrolytic production, it was discovered that oxalic acid precipitation method has more advantages such as high electrolytic efficiency and low impurity compared with the ammonium bicarbonate precipitation method, which is a consensus in rare earth electrolytic industry. Due to the higher production cost of oxalic acid, ammonium bicarbonate has become the main raw material for Pr-Nd oxide electrolysis. And it was found that there is no significant difference of chemical properties between Pr-Nd oxides prepared by the two methods. Therefore, the influence of physical properties of $\mathrm{Pr}-\mathrm{Nd}$ oxide on its electrolysis was studied in this paper.

At present, the quality requirements of molten salt electrolysis of Pr-Nd oxide is only limited to chemical properties, which mainly include the content of rare earth and impurity ions of Pr-Nd oxide. In the actual electrolysis process, it is also required that $\mathrm{Pr}-\mathrm{Nd}$ oxides should have characteristics of no dust, less precipitation at the bottom, good fluidity and fast dissolving rate in the electrolyte, so as to ensure the normal electrolytic process. However, these properties are mainly determined by physical properties of Pr-Nd oxide, such as the specific surface area, particle size, bulk density, morphology, zeta potential and mobility, etc.

\subsection{Effect Mechanism of Physical Properties of Pr-Nd Oxide on the Electrolysis}

The electrolysis process of rare earth is that rare earth oxides are decomposed into rare earth cations and oxygen anions in molten fluoride. Discharge reactions occur respectively on the cathode and anode under direct current. Chemical reactions are as follows:

$$
\begin{aligned}
& \text { Dissolution reaction: } \mathrm{RE}_{2} \mathrm{O}_{3}=2 \mathrm{RE}^{3+}+3 \mathrm{O}^{2-} \\
& \text { Cathodic reaction: } \mathrm{RE}^{3+}+3 \mathrm{e}=\mathrm{RE} \\
& \text { Anodic reaction: } 2 \mathrm{O}^{2-}+\mathrm{C}-4 \mathrm{e}=\mathrm{CO}_{2} \\
& \text { Total reaction: } \mathrm{RE}_{2} \mathrm{O}_{3(\mathrm{~s})}+3 / 2 \mathrm{C}_{(\mathrm{s})}=2 \mathrm{RE}_{(\mathrm{l})}+3 / 2 \mathrm{CO}_{2(\mathrm{~g})}
\end{aligned}
$$

From the reactions above, it was found that Pr-Nd oxide is dissolved and dissociated into ions in the molten salt electrolyte first, then these ions are discharged and precipitated to form metal on the cathode. Therefore, it can be concluded that physical properties of rare earth oxides mainly affect its solubility in the molten salt electrolyte. In the actual industrial electrolytic cell, the small Pr-Nd oxide particles produce a lot of dust which cause material losses. As a result, these particles float on the surface of the electrolyte solution. At the same time, it is difficult for these particles to dissolve with the boiling electrolyte. As a result, the viscosity of electrolyte increases, which makes not only the separation of carbon residue more difficult, but also decreases current efficiency significantly. On the contrary, large Pr-Nd oxide particles cannot be dissolved immediately and take longer time to dissociate. Some of them even not been fully dissolved after next feeding. These insoluble Pr-Nd oxide particles will sink directly into the bottom of electrolytic cell and cause sticky crusts. After a long period of time, the internal structure of electrolytic cell will be changed to form a biased flow 
phenomenon, this phenomenon leads to decrease of the current efficiency ultimately. Therefore, either the Pr-Nd oxide particles float on the surface or sink at the bottom of the molten salt electrolyte are not suitable for electrolysis. The appropriate particle size of raw material is easier to electrolyze in molten salt. It shows that physical properties of $\mathrm{Pr}-\mathrm{Nd}$ oxide significantly affect the molten salt electrolysis process.

\subsection{Requirements for Physical Properties of Pr-Nd Oxide in Molten Salt Electrolysis Process}

The physical properties of Pr-Nd oxide were compared and analyzed, which are produced by 8 long-term producers for an anonymous electrolytic company. The characteristics of physical properties of these raw materials were studied, which mainly affect dissolution rate of Pr-Nd oxide in the molten salt electrolyte; however, there is no mature and reliable method to study on dissolution rate of rare earth oxides in molten salt. As a result, hydrochloric acid solution is used as the reference of molten salt system to study the relationship between the physical properties of $\mathrm{Pr}-\mathrm{Nd}$ oxide and its dissolution rate in hydrochloric acid solution, which can be analogized as the relation of $\mathrm{Pr}-\mathrm{Nd}$ oxide in molten salt. Then suitable range of physical properties of the electrolytic Pr-Nd oxide was determined and the relevant results were verified by industrialized operation. Table 1 lists the relationship between the range of physical properties of the raw materials of different producers and its acid dissolution rate.

Table 1. Relationship between the range of physical properties of Pr-Nd oxide of different producers and its acid dissolution rate.

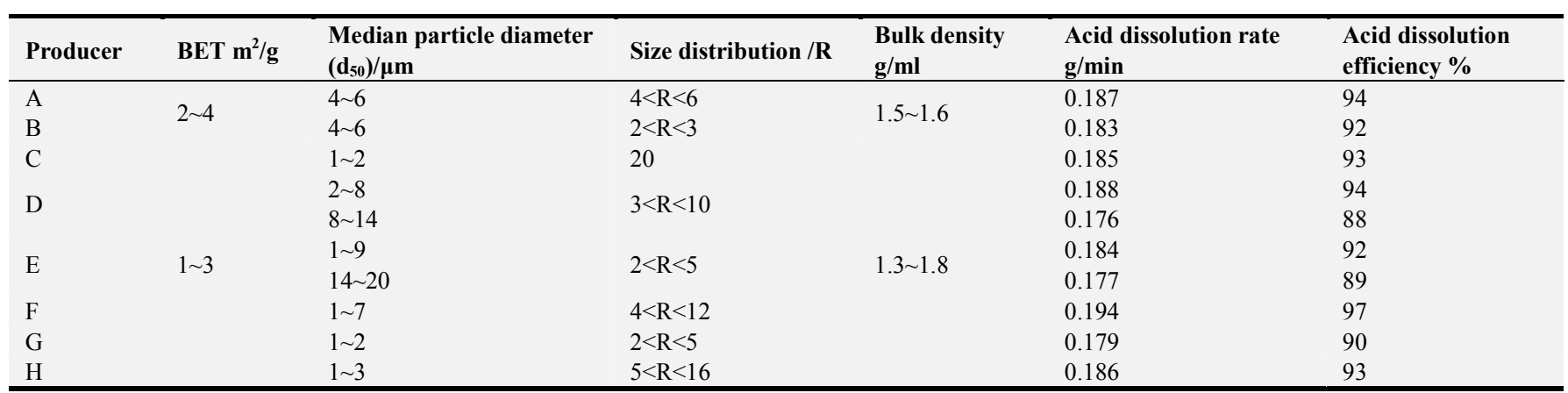

Note: Producers of A and B use the oxalic acid precipitation method;

Producers of $\mathrm{C}$ to $\mathrm{H}$ use the ammonium bicarbonate precipitation method.

\subsubsection{Particle Size}

It can be seen from table 1 , the median particle size $\mathrm{d}_{50}$ of $\mathrm{A}$ and $\mathrm{B}$ producers is in the range of 4 to 6 microns and the size distribution is more uniform, and their acid dissolution efficiencies are above $90 \%$. While compared with, the particle size of raw materials prepared by $\mathrm{C}$ to $\mathrm{H}$ producers using the oxalic acid precipitation method mainly distributes between 1 to 20 microns, the particle size range is broaderand the size distribution is not uniform enough. For both $\mathrm{D}$ and $\mathrm{E}$ producers, the raw materials' median particle size $d_{50}$ concentrates in two regions. For the D producer, the particle size $d_{50}$ is concentrated in the range of $2 \sim 8 \mu \mathrm{m}$ and $8 \sim 14 \mu \mathrm{m}$ respectively, and the acid dissolution efficiencies are $94 \%$ and $88 \%$. For the E producer, the particle size $\mathrm{d}_{50}$ is concentrated in the range of $1 \sim 9 \mu \mathrm{m}$ and $14 \sim 20 \mu \mathrm{m}$ respectively, and the acid dissolution efficiencies are $92 \%$ and $89 \%$. Median particle sizes $\mathrm{d}_{50}$ of raw materials from the rest producers are all below $10 \mu \mathrm{m}$, and the acid dissolution efficiencies remained beyond $90 \%$. When the median particle size $\mathrm{d}_{50}$ is between 1 to $10 \mu \mathrm{m}$, the acid dissolution efficiency of raw material is above $90 \%$. On the contrary, when $\mathrm{d}_{50}>10 \mu \mathrm{m}$, the acid dissolution efficiency is below $90 \%$. It indicates the smaller the particle size is, the higher the acid dissolution efficiency is, and materials with small particles $\left(1 \mu \mathrm{m}<\mathrm{d}_{50}<10 \mu \mathrm{m}\right)$ prepared by both oxalic acid and ammonium bicarbonate precipitation methods have higher dissolution rates. On the other hand, that with large particles $\quad\left(\mathrm{d}_{50}>10 \mu \mathrm{m}\right)$ prepared by the ammonium bicarbonate precipitation method will lead to lower dissolution rate relatively. Moreover, practical production requires the particle size of $\mathrm{Pr}-\mathrm{Nd}$ oxide is suitable for electrolytic process, so that oxalic acid precipitation method is more applicable to the molten salt electrolysis.

\subsubsection{Specific Surface Area}

The larger the specific surface area of Pr-Nd oxide, the smaller the particle size is, and the surface tension decreases, which enhances the wettability between the electrolyte and Pr-Nd oxide. Under the same precipitation processing conditions, it indicates the higher calcining intensity and crystallinity. Meanwhile, dissolution rate of Pr-Nd oxide in electrolyte decreases considerably. According to the table 1, the specific surface area of Pr-Nd oxide particles produced by oxalic acid precipitation method is larger than that by ammonium bicarbonate precipitation method, and the specific surface area is mainly concentrated in the range of $2 \sim 4 \mathrm{~m}^{2} / \mathrm{g}$ and the acid dissolution efficiency remains above $90 \%$. While, the specific surface area of raw materials produced by ammonium bicarbonate precipitation is $1 \sim 3 \mathrm{~m}^{2} / \mathrm{g}$ and the acid dissolution efficiency is unstable. Therefore, oxalic acid precipitation method produces particles with larger specific surface area, smaller and more uniform size. The wettability between electrolyte and Pr-Nd oxide is better, and thus the efficiency of the acid precipitation method is higher than the ammonium bicarbonate method. 


\subsubsection{Bulk Density}

According to the table 1 , the bulk density of A and B produced Pr-Nd oxide particles is mostly concentrated in $1.5 \sim$ $1.6 \mathrm{~g} / \mathrm{mL}$, and for $\mathrm{C}$ to $\mathrm{H}$ produced particles is $1.3 \sim 1.8 \mathrm{~g} / \mathrm{ml}$. Combined with 3.3 .1 , the bulk density is affected by particle size. The bulking density of raw materials produced by oxalic acid precipitation method is relatively stable due to their stable particle size. On the other hand, that by the ammonium bicarbonate precipitation method has wider particle size distribution, which leads to a wider range of bulk density. Furthermore, the physical properties of Pr-Nd oxide particles produced by the oxalic acid precipitation is more stable.

\subsubsection{Microstructure}
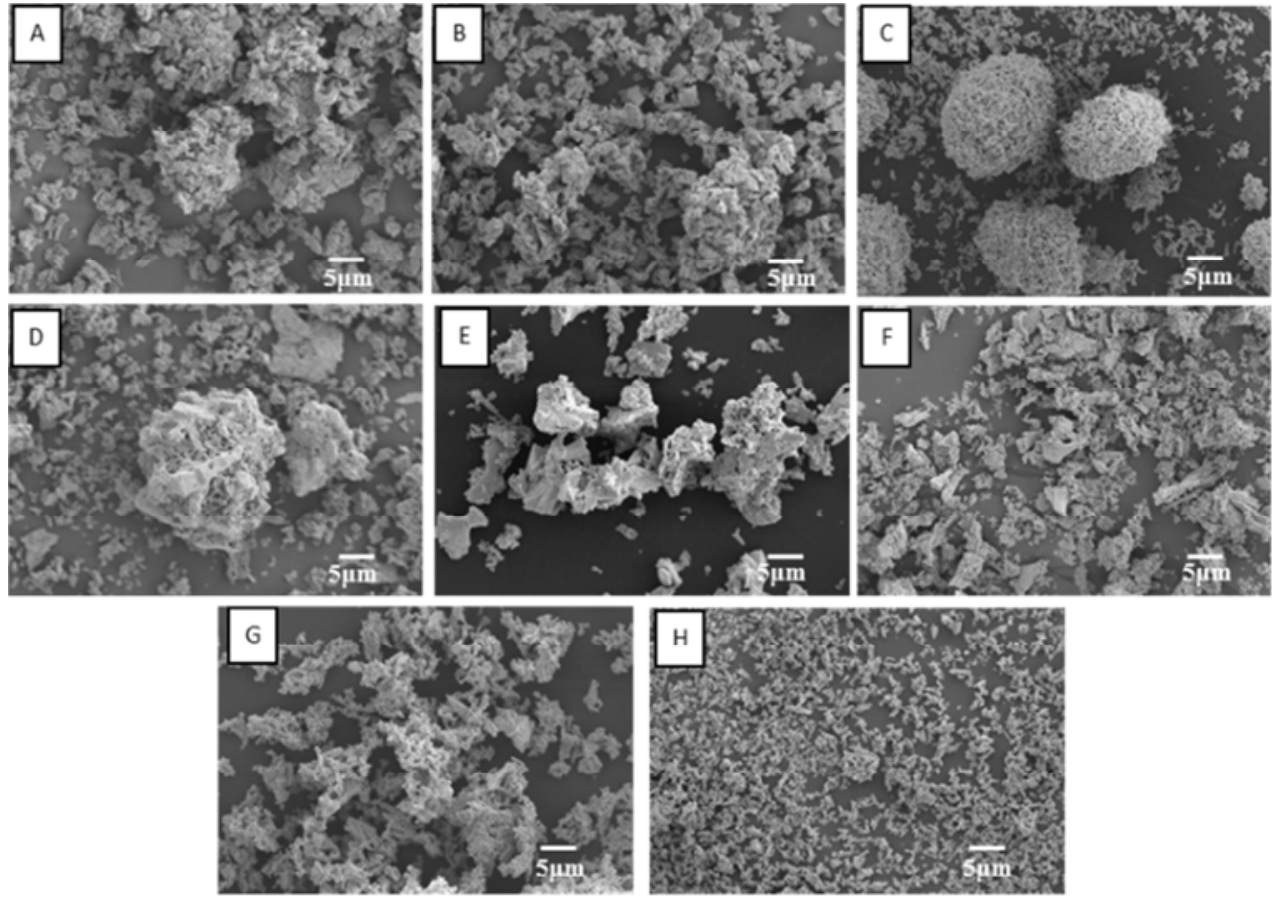

Figure 1. Scanning electron microscope images of $\mathrm{Pr}$-Nd oxide from different producers.

Figure 1 gives the SEM images of $\mathrm{Pr}-\mathrm{Nd}$ oxide from different producers. The results present that the morphology of $\mathrm{A}$ and $\mathrm{B}$ are loose, porous and irregular aggregation. The differences from $\mathrm{C}$ to $\mathrm{H}$ are fairly enormous. $\mathrm{C}$ contains fluffy spheres and loose pellets, D looks like irregular spheres, E to $\mathrm{G}$ forms irregular aggregates. $\mathrm{G}$ has porous structure on its surface, which is similar as particles produced by oxalic acid precipitation. $\mathrm{H}$ looks like dispersed and loose pellets. Combine with 3.3.1 and 3.3.2, it is concluded that the raw materials produced by oxalic acid precipitation method have larger specific surface area, smaller and more uniform particle size, porous structure on its surface and no obvious melting phenomenon. Therefore, Pr-Nd oxide particles could be dissolved sufficiently and rapidly in the process of molten salt electrolysis.

\subsection{Production Verification of Electrolysis}

Two batches of totally 5 tons Pr-Nd oxide raw material were purchased from E company, which were assigned as 1\# and 2\#. Both were processed in the no. 2 stove of departments $D$ in a RE electrolyze company for 11days, then was supervised by special workers. The Chemical and physical properties are mainly assessed in lab to confirm whether the chemical analogy experiment can be used as the reference of molten salt electrolysis experiments, thus to determine suitable range of physical properties of $\mathrm{Pr}-\mathrm{Nd}$ oxide.

\subsubsection{Assessment of Chemical Properties}

Table 2. Chemical composition analysis of the Pr-Nd oxide.

\begin{tabular}{|c|c|c|c|c|c|c|c|c|c|c|c|c|}
\hline NO. & REO & $\begin{array}{l}\text { Burning } \\
\text { loss }\end{array}$ & $\mathrm{Cl}^{-}$ & $\mathrm{SO}_{4}{ }^{2-}$ & $\mathrm{SiO}_{2}$ & $\mathrm{Fe}_{2} \mathrm{O}_{3}$ & $\mathrm{CaO}$ & $\mathrm{Nd}_{2} \mathrm{O}_{3}$ & $\operatorname{Pr}_{6} \mathbf{O}_{11}$ & $\begin{array}{l}\text { Remain } \\
\text { RE }\end{array}$ & $\begin{array}{l}\text { Not } \\
\text { dissolved }\end{array}$ & $\mathrm{Na}_{2} \mathrm{O}$ \\
\hline $1 \#$ & 99.57 & 0.1 & 0.012 & 0.012 & 0.01 & 0.0011 & 0.0071 & 73.55 & 26.4 & $<0.01$ & 0.017 & 0.027 \\
\hline $2 \#$ & 99.57 & 0.1 & 0.019 & 0.012 & 0.01 & 0.0013 & 0.0072 & 74.79 & 25.16 & $<0.01$ & 0.011 & 0.029 \\
\hline
\end{tabular}

From table 2, Chemical composition of two batches Pr-Nd oxide produced by E company meet the requirements of electrolysis, except for the relatively high content of impurities $\mathrm{Na}_{2} \mathrm{O}$. In practical production, both batches of raw materials produce large amount of smoke when feeding. The two batches of raw materials are produced by the sodium 
carbonate precipitation method, thus the $\mathrm{Na}$ content of raw materials is much higher than that produced by ammonium bicarbonate precipitation. During the washing process, only $\mathrm{NaCl}$ adsorbed on the surface of the $\mathrm{PrCO}_{3} \quad\left(\mathrm{NdCO}_{3}\right)$ precipitation is washed off, however, the $\mathrm{NaCl}$ wrapped inside of the precipitation cannot be extracted and will not evaporate as the $\mathrm{PrCO}_{3}\left(\mathrm{NdCO}_{3}\right)$ decomposing in the calcination process, which eventually leads to the enrichment of $\mathrm{Na}^{+}$in the $\mathrm{Pr}-\mathrm{Nd}$ oxide, producing much smoke.

\subsubsection{Assessments of Physical and Dissolution Properties}

Table 3 presents the physical and dissolution properties of the two batches Pr-Nd oxide particles. According to the table, the particle size $d_{50}$ is in the range of $10 \sim 20 \mu \mathrm{m}$ and relatively uniform, the bulk density remains $1.7 \sim 2.0 \mathrm{~g} / \mathrm{mL}$, and the acid dissolution efficiency is below $90 \%$. Combined with 3.3.1, when $\mathrm{d}_{50}>10 \mu \mathrm{m}$, the rate of acid dissolution is low. Base on the relationship between fluidity and the rest angle, the fluidity of $2 \#$ is far better than that of the $1 \#$ (When angle $<40^{\circ}$, fluidity is good; when angle is between $40^{\circ}$ and $60^{\circ}$, the fluidity is ordinary, when angle $>60^{\circ}$, the fluidity is bad).

Figure 2 shows the scanning electron microscope (SEM) images of the two batches of materials. It can be seen that in microstructure, though irregular groups are formed during high temperature calcination, quite a lot of pores form on the surface of the $1 \#$ materials. However, the morphology of the 2\# materials is formed by a great number of sphere pellets which are completely melted on the surface. Combined with 3.3.4 and the table 3, it can be seen that there is pore structure and no obvious melting phenomenon on the micro surface, which is good for fast and complete dissolution of Pr-Nd oxide during electrolysis process, therefore, the acid dissolution rate of $1 \#$ materials is much better than that of $2 \#$.

Table 3. Results of physical and dissolution properties of raw material from E company.

\begin{tabular}{|c|c|c|c|c|c|c|c|c|}
\hline \multirow{2}{*}{ No. } & \multicolumn{3}{|c|}{ Partial size / $\mu \mathrm{m}$} & \multirow{2}{*}{$\begin{array}{l}\text { Partial size } \\
\text { distribution / R }\end{array}$} & \multirow{2}{*}{$\begin{array}{l}\text { Fluidity (rest } \\
\text { angle) }\end{array}$} & \multirow{2}{*}{$\begin{array}{l}\text { Bulk density } \\
/ \mathrm{g} / \mathrm{mL}\end{array}$} & \multirow{2}{*}{$\begin{array}{l}\text { Rate of acid } \\
\text { dissolution } / \mathrm{g} / \mathrm{min}\end{array}$} & \multirow{2}{*}{$\begin{array}{l}\text { Efficiency of acid } \\
\text { dissolution } / \%\end{array}$} \\
\hline & $d_{10}$ & $d_{50}$ & $d_{90}$ & & & & & \\
\hline $1 \#$ & 1.073 & 19.48 & 45.71 & 2.3 & $61^{\circ}$ & 1.792 & 0.175 & 88 \\
\hline $2 \#$ & 0.748 & 15.92 & 38.61 & 2.4 & $50^{\circ}$ & 2.004 & 0.133 & 67 \\
\hline
\end{tabular}
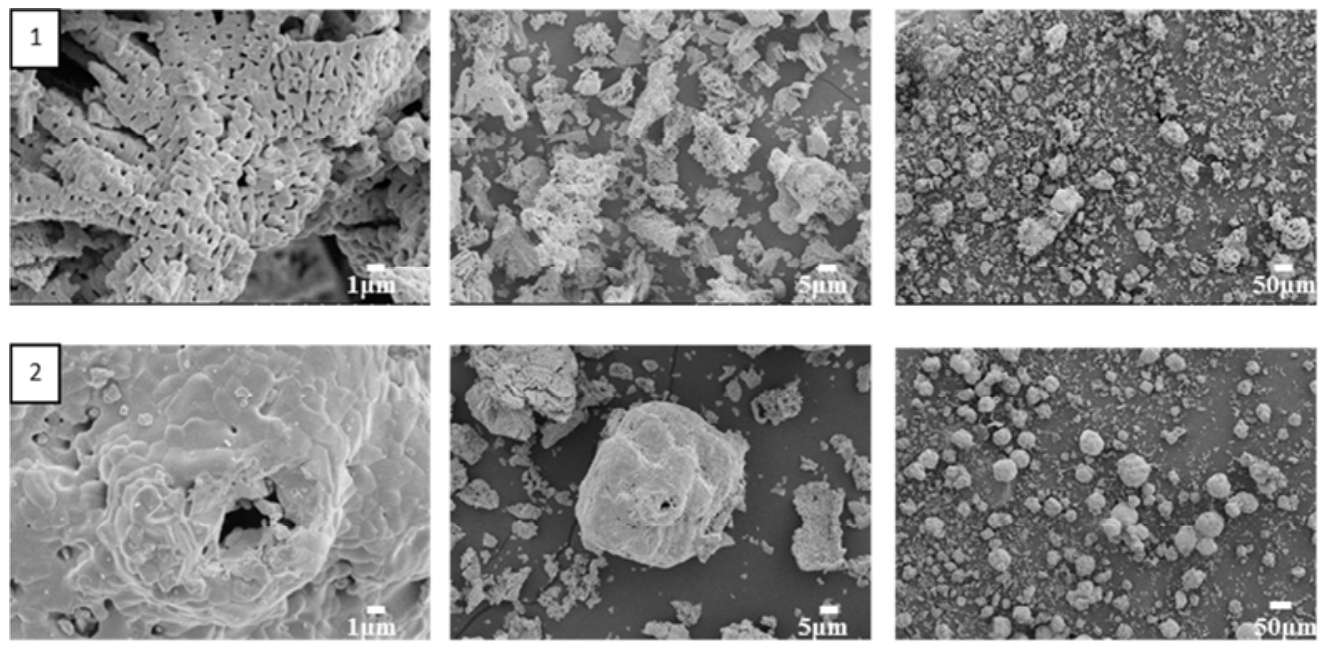

Figure 2. Scanning electoral Microscope (SEM) of materials from E company.

\subsubsection{Electrolysis Production}

Table 4. Quality criteria parameters of 61 stove before using E company's product.

\begin{tabular}{|c|c|c|c|c|c|}
\hline Stove NO. & $\mathrm{C}$ content $/ \%$ & Fe content $/ \%$ & Feeding amount/kg & Output/kg & Metal lose /kg \\
\hline D275 & 0.03 & 0.23 & 90 & 80 & 1.7 \\
\hline D276 & 0.03 & 0.25 & 85 & 82 & 8.05 \\
\hline D277 & 0.032 & 0.25 & 85 & 75 & 1.05 \\
\hline D278 & 0.015 & 0.1 & 85 & 74 & 0.05 \\
\hline D279 & 0.03 & 0.23 & 85 & 74 & 0.05 \\
\hline$\ldots$ & $\ldots$ & $\ldots$ & $\ldots$ & $\ldots$ & $\ldots$ \\
\hline D331 & 0.021 & 0.14 & 80 & 66 & -0.6 \\
\hline D333 & 0.021 & 0.13 & 80 & 73 & 3.4 \\
\hline D334 & 0.014 & 0.21 & 85 & 72 & 0.05 \\
\hline D335 & 0.016 & 0.18 & 75 & 65 & -0.25 \\
\hline Average & 0.024 & 0.20 & 82.8 & 71.4 & -0.12 \\
\hline Total & / & / & 5050 & 4353 & Ratio:1.208 \\
\hline \multicolumn{5}{|c|}{$\mathrm{Na}$ deposition in stove } & 0.25 \\
\hline
\end{tabular}


Table 5. Criteria quality parameters of 61 stove after using $E$ company's product.

\begin{tabular}{llllll}
\hline Stove NO. & C content/\% & Fe content/\% & Feeding amount/kg & Output/kg & Metal lose $/ \mathbf{k g}$ \\
\hline D336 & 0.015 & 0.15 & 90 & 74 & -4.3 \\
D337 & 0.037 & 0.19 & 85 & 81 & 7.05 \\
D338 & 0.036 & 0.21 & 90 & 79 & 0.7 \\
D339 & 0.037 & 0.18 & 85 & 70 & -3.95 \\
D340 & 0.036 & 0.19 & 85 & 65 & -8.95 \\
$\ldots \ldots$ & $\ldots \ldots$ & $\ldots \ldots$ & $\ldots \ldots$ & $\ldots \ldots$. & 0.4 \\
D392 & 0.012 & 0.15 & 80 & 70 & 6.4 \\
D393 & 0.011 & 0.15 & 80 & 76 & 4.05 \\
D394 & 0.014 & 0.11 & 85 & 77 & -0.95 \\
D395 & 0.016 & 0.15 & 85 & 73 & -10.95 \\
D396 & 0.011 & 0.19 & 83 & 63 & -0.52 \\
Average & 0.021 & $/$ & 5065 & 71.4 & -31.65 \\
Total & $/$ & & & 4356.4 & 0.58 \\
Na deposition in stove & & & & \\
\hline
\end{tabular}

Tables 4 and 5 present the quality criteria parameters of 61 stove before and after using E company's product. In the tables, the $\mathrm{C}$ and Fe content of Pr-Nd metal in stove D didn't change obviously, and both are qualified to the electrolysis process before and after using by E company's product. While $\mathrm{Na}$ accumulated enormously in stove after using E company's product, and its content increased from $0.25 \%$ to $0.58 \%$. The two batches of raw material were processed by stove, which started from NO. 336 and ended on NO. 396, totally 61 stoves of outputs were produced. $5065 \mathrm{~kg}$ Pr-Nd oxide and $300 \mathrm{~kg}$ Pr-Nd fluoride were consumed. The direct ratio is 1.163 , and total ratio is 1.221 . The loss of metal is $31.65 \mathrm{~kg}$ after using E company's product. Compared to previous process, the total ratio increases 0.013 , total loss increases $24.15 \mathrm{~kg}$. Thus, the yield of the two batches products were both low, and also the accumulated $\mathrm{Na}$ affects the efficiency of electrolysis. Moreover, associated with 3.4.2, the acid dissolution rate of the two batches Pr-Nd oxide prepared by sodium carbonate precipitation method is only $78 \%$, about $22 \%$ of the Pr-Nd oxide didn't dissolute within the valid time, and the acid dissolution rate is lower than $90 \%$. Consequently, the acid dissolution rate of $\mathrm{Pr}-\mathrm{Nd}$ in hydrochloric acid could refer to its dissolution rate in molten salt. Thus, hydrochloric acid can be used as the reference of the molten salt. $\mathrm{Na}$ accumulation in the electrolysis process affects the electrolysis efficiency.

\section{Conclusions}

The physical properties of Pr-Nd oxide prepared by oxalic acid precipitation are more stable than that prepared by ammonium bicarbonate precipitation method. The stability could reduce the electrolyze cell risk in the process of material alternation. In addition, it also affects the electrolysis efficiency and the product quality. It can be concluded that the oxalic acid precipitation is more suitable for molten salt electrolysis, which can be considered as the standard of ammonium bicarbonate precipitation.

The particle size, specific surface area, morphology, bulk density and fluidity are significant criteria parameters of the Pr-Nd oxide. Also, particle size and morphology are the key factors. It is required that the particle size of Pr-Nd oxide with good physical property is uniform and not too big or small. Additionally, the microstructure should not over disperse, but with agglomerate status, besides the micro surface should have porous structure to avoid melting phenomenon.

\section{References}

[1] Zhang H, Huang J Y, Ban D S. Discussion on Influence of Alumina Physical Property on Aluminum Electrolysis Industry. Nonferrous Metallurgy energy saving, 2012, 8(4):15.

[2] Yang F. Influence of Alumina Quality on Aluminum Electrolysis and its Control Analysis. Low Carbon World, 2016, 6: 257.

[3] Yang G W, Yang G F. Effect of Alumina Powder on Aluminum Electrolysis. Metal World, 2016, 6: 28.

[4] Lu X C, Tang H H. Improvement of Alumina Quality and its Effect on The Aluminum Smelting. China Nonferrous Metallurgy, 2006, 8(4): 14.

[5] Zhang W M, Zhuang J X, Chen Q. Cost-Effective Production of Pure $\mathrm{Al}_{13}$ from $\mathrm{AlCl}_{3}$ by Electrolysis. Industrial \& Engineering Chemistry Research, 2012, 51:11201.

[6] Zhang W M, Zhuang J X, Chen Q, et al. A More Ecofriendly Cold Ramming Paste for an Aluminum Electrolysis Cell with Phenol-Formaldehyde Resin As Binder. Industrial \& Engineering Chemistry Research, 2013, 52: 1750.

[7] Wang S N, Duan Z B, Hong Y X. Effect of Alumina Quality on Aluminum Electrolysis Process. Jiuquan Iron and Steel Technology, 2015, 2: 18.

[8] Zhang J W, Chen Y L. Effect of Particle Size and Concentration of Alumina on Electrolytic Production. Xinjiang Nonferrous Metals, 2010, 95.

[9] Li H Q, Liu H W, Zhang R X, et al. Effects of Calcination Temperature on Specific Surface Area and Density of Pr-Nd Binary Oxides. Rare Earths, 2010, 31(6): 78-80.

[10] Zhang R X, Li H q, Liu H W, et al. Effects of Calcination Temperature on Particle Size and Morphology of $\mathrm{Pr}-\mathrm{Nd}$ Oxides. Rare Earths, 2010, 31(1):48.

[11] Zhao W Y, Xu Yan H, Li Z Q, et al. Effect of Preparation Conditions on The Physical Properties of Pr-Nd Oxides. Rare Earths, 2015, 36(6):111.

[12] Liu Y L, Nie Z W, Luo Q S, et al. Effects of Physical Properties of Pr-Nd Oxide on its Electrolysis Preparation and Improvement Measures. Rare Metals and Cemented Carbides, 2016, 44(2): 50. 